\title{
Simplified method of grading primary carcinomas of the breast
}

\author{
D M Parham, N Hagen, R A Brown
}

\begin{abstract}
Aims: To produce a simplified prognostic index for breast cancer.

Methods: A retrospective study of 105 cases of primary infiltrating breast adenocarcinoma (not otherwise specified) was performed. Mitotic counts and semiquantitative assessment of tumour necrosis, fibrosis tubule formation, nuclear pleomorphism were made in histological sections of the primary tumour. Statistical analysis was performed to determine which of these parameters best predicts the observed survival.

Results: Mitotic count and necrosis correlated best with survival. This allowed the formation of a simple prognostic index based on these two parameters.

Conclusions: This new prognostic index, with four tumour grades, seemed to be superior to Bloom's grading method, with greater separation of the prognostic groups. In particular, there is clear segregation of a group of patients with a distinctly poor prognosis.
\end{abstract}

One of the most challenging problems in breast cancer is determining the likely prognosis of a patient's survival. Although a proportion $(25 \%)$ of primary breast tumours, termed special types, ${ }^{1}$ have easily recognisable histological features and are associated with a good prognosis, most $(75 \%)$ have no distinguishing features and are termed infiltrating ductal carcinomas (not otherwise specified). In this latter group tumours with apparently similar histological features have wildly different survival times ranging from a few months to many decades. This makes it difficult to predict prognosis.

The degree of tumour differentiation is an important prognostic factor. Von Hansemann is credited with initiating many of the grading systems used to assess malignant neoplasms. ${ }^{2}$ Greenough $^{3}$ applied this to breast cancer, developing three histological grades of malignancy based on tubule formation, secretory activity of the cells, the overall size of the cells and nuclei, variation in the size of both the cells and nuclei, nuclear hyperchromatism, and mitotic counts. ${ }^{3}$ Patey and Scarff followed Greenough's method but gave chief importance to tubule formation, variation in nuclear size and shape, and nuclear hyperchromatism. ${ }^{4}$ In 1957 Bloom and Richardson revitalised the grading of breast cancer, making it more acceptable by introducing a numerical scoring system based on three features: tubule formation, nuclear pleomorphism and the frequency of nuclear hyperchromatism, and mitotic figures. ${ }^{5}$ In recent years Elston has refined the definition and method of assessing the parameters which constitute Bloom's grading method and this is currently the most widely used method of grading breast tumours. ${ }^{6}$

We have recently shown that extensive tumour necrosis is an indicator of poor outcome, ${ }^{7}$ confirming the not widely appreciated findings of both Fisher et $a l^{8}$ and Dixon et al. ${ }^{9}$ In this study we objectively evaluated the potential of using tumour necrosis or fibrosis as prognostic factors, with the aim of developing a simpler prognostic method of grading primary tumours. As part of the study we also statistically evaluated the relative contribution to survival of each of the three components (tubule formation, nuclear pleomorphism, and mitotic count) used in Bloom's method of grading.

\section{Methods}

This study is based on data from 105 women with primary infiltrating ductal adenocarcinoma (not otherwise specified) of the breast, diagnosed in 1980 and 1981 and registered with Tayside Cancer Registry. All currently recognised special types of breast cancer were excluded. ${ }^{10}$ Follow up for the purposes of this study stopped in December 1990. Information regarding menstrual status was generally not available. If fine needle aspiration biopsy had been performed this was recorded. The mean patient age was 59 years (median 59 years, range 30-94 years). Surgical treatment consisted of simple mastectomy and radiotherapy (84 cases), or wedge resection and radiotherapy ( 21 cases). At the end of the follow up period 57 patients had died and the survival time in each case was recorded.

The original haematoxylin and eosin stained sections were retrieved from the files and examined by standard light microscopy without knowledge of the patients' details. The tumours were assessed on a double headed microscope by two observers-one pathologist with a special interest in breast disease (DMP), the other a trainee pathologist $(\mathrm{NH})$. Where variance between the observers occurred on a particular feature, a consensus decision was reached by discussion.

A detailed description of the features used to assess Bloom's grade has been described by Elston. ${ }^{46}$ Briefly, the following aspects of the primary tumour were graded on a three point 
scale: tubule formation, mitotic count, and nuclear size or pleomorphism. Mitotic counts were performed at the growing edge of the tumour at $\times 400$ (field diameter $0.45 \mathrm{~mm}$ ) and only structures which could not be misinterpretated as anything but mitotic figures were counted. For each tumour a score between 3 and 9 would be obtained. Tumours scoring 3 to 5 formed Bloom's grade 1 (well differentiated), 6-7 formed Bloom's grade 2 (moderately differentiated), and 8-9 Bloom's grade 3 (poorly differentiated).

Tumour fibrosis and necrosis were each assessed on a four point scale: $0=$ none present, $1=$ necrosis or fibrosis occupying up to $\frac{1}{2}$ a low power field (maximum diameter less than $2.28 \mathrm{~mm}$ ), $2=\frac{1}{2}$ to one low power field $(2 \cdot 28-4.57 \mathrm{~mm}), 3=$ greater than one low power field (maximum diameter greater than $4.57 \mathrm{~mm}$ ). When multiple areas of necrosis or fibrosis were present only the largest focus was assessed. The minimal positive evidence of necrosis was a cluster of four necrotic cells exhibiting either apoptosis with cell fragments comprising pyknotic chromatin and eosinophilic cytoplasm, or coagulative necrosis where the cell outline is maintained but with altered cytoplasmic staining and nuclear pyknosis, kaaryolysis, or karyohexis.

Cox's regression analysis, ${ }^{10}$ which uses a proportional hazards model, was performed on an IBM compatible personnel computer using CSS software (Statsoft, USA). Briefly, the hazard at any time is the risk of dying at that time and Cox's regression analysis is a numerical method of relating hazard to the values of factors which are assumed to be of prognostic value. This is done by constructing a multiple linear relation between the natural logarithm of the hazard and the factors of interest. The coefficients $(\beta \mathrm{s})$ in the linear relation quantify the effect of the corresponding factors; a positive value for a coefficient indicates that an increase in the value of the corresponding factor results in an increased hazard and the larger the coefficient is, the greater the effect of a given increase in the value of that factor. Conversely, a negative coefficient would indicate a factor for which an increase in value would result in a decrease in hazard.

The CSS software also produces the standard errors of the coefficients and the corressponding $t$ values, which are the coefficient values divided by their standard errors. In interpreting the results of the analysis we have used the recognised convention that a coefficient is regarded as significant at the $\mathbf{5 \%}$ level if the corresponding $t$ value is greater than 1.96 .

\section{Results}

Analysis of the data showed that there were 23 Bloom's grade 1 tumours, 48 grade 2, and 34 grade 3 . The survival curves for each of these groups of tumours is shown in fig 1A. The results of Cox regression of all the variables studied are shown in table 1 . This shows that of the three components of Bloom's grading,
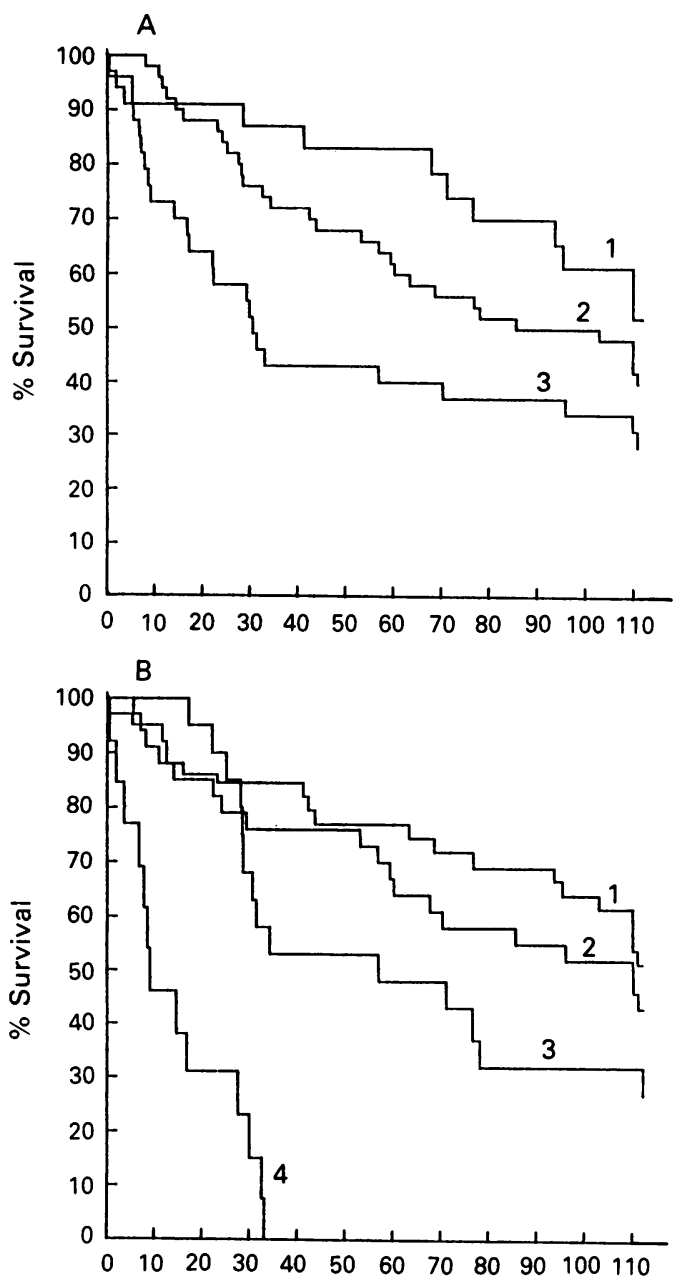

Figure 1 Survival curves for Bloom's grade $(A)$ and the new prognostic grades (B).

mitotic count contributes most to the prediction of survival.

Two variables (table 1) were selected as having an important influence on survival: mitotic count and necrosis. The other variables have no major influence on prognosis. No correlation was found between necrosis and a history of fine needle aspiration biopsy (results not shown). Re-evaluation of mitosis and necrosis alone by Cox's analysis permits refinement of the coefficient with the following values for mitoses and necrosis respectively; $\beta$ 0.379 ( $t$ value 2.378 ) and 0.610 ( $t$ value 4.957). These coefficients can be used to form a prognostic index:

$$
\begin{gathered}
\text { Prognostic index }= \\
(0.38 \times \text { Mitosis })+(0.61 \times \text { Necrosis })
\end{gathered}
$$

The index quantifies the relation beween the hazard and the mitotic score and degree of necrosis because the natural logarithm of the hazard at any time is proportional to:

$$
0.38 \times \text { mitosis }+0.61 \times \text { necrosis }
$$

Recalling that hazard is the probability of dying at any specified time, we see that the hazard at any time is proportional to:

$$
\mathrm{e}^{0.38 \times \text { mitosis } \times 0.61 \times \text { necrosis }}
$$

The implication of this may be made clearer by considering the change in hazard which results from an increase by one point in the score for mitosis or necrosis. The effect of an increase of 
Table $1 \quad \beta$ and $t$ values obtained by Cox's analysis for each of the variables assessed in 105 breast tumours

\begin{tabular}{|c|c|c|c|}
\hline Variable & $\beta$ & $\begin{array}{l}\text { Standard } \\
\text { error }\end{array}$ & $t$ value \\
\hline $\begin{array}{l}\text { Tubule formation } \\
\text { Nuclear } \\
\text { pleomorphism }\end{array}$ & $\begin{array}{r}0.142 \\
-0.006\end{array}$ & $\begin{array}{l}0.225 \\
0.272\end{array}$ & $\begin{array}{r}0.633 \\
-0.022\end{array}$ \\
\hline $\begin{array}{l}\text { Mitotic count } \\
\text { Necrosis } \\
\text { Fibrosis }\end{array}$ & $\begin{array}{l}0.353 \\
0.604 \\
0.028\end{array}$ & $\begin{array}{l}0 \cdot 178 \\
0 \cdot 126 \\
0 \cdot 144\end{array}$ & $\begin{array}{l}1 \cdot 976 \\
4 \cdot 807 \\
0 \cdot 193\end{array}$ \\
\hline
\end{tabular}

a score of 1 in mitosis is $\mathrm{e}^{0.38 \text { or } 1.46}$. This implies that at any particular time a patient with a mitosis score of 2 is 1.46 times more likely to die shortly thereafter compared with a patient with a tumour mitosis score of 1 , provided that their necrosis scores are the same. A similar calculation for a change of one point in necrosis implies that a patient is $1.84\left(\mathrm{e}^{0.61}\right)$ times more likely to die as a patient with a tumour with no necrosis, provided that their mitoses scores are the same. As the hazards are proportional a patient with a mitosis score of 3 is $1.46 \times 1.46=2.13$ times as likely to die shortly after as a patient with a tumour mitosis score of 1 . Similarly, a patient with a tumour mitosis score of 2 and a necrosis score of 1 is $1.46 \times 1.84=2.69$ times as likely to die in a short interval thereafter as a patient with a mitosis score of 1 point and necrosis score of 0 points. The complete set of possibilities and the way they correspond to increase the risk of death relative to a patient with a tumour mitosis of score 1 and a necrosis score of 0 is summarised in table 2 .

For each tumour one of 12 possible combined scores was obtained by combining one of three values for mitosis and one of four values for necrosis (table 3). For each prognostic index score a computer generated survival curve was obtained. Empirical evaluation of each of these survival curves suggested allocating the scores into one of four groups (grades):

Prognostic index grade 1 PIG 1 (39 cases) tumours scoring 0.38, PIG 2 (34

Table 2 Estimated proportionate change in hazard for all combinations of mitotic counts and degrees of necrosis compared with a tumour mitosis 1 and necrosis 0

\begin{tabular}{llllr}
\hline \multirow{5}{*}{ Mitosis } & \multicolumn{4}{l}{ Necrosis } \\
\cline { 2 - 5 } & 0 & 1 & 2 & \multicolumn{1}{l}{3} \\
\hline 1 & 1.00 & 1.84 & 3.39 & 6.23 \\
2 & 1.46 & 2.69 & 4.95 & 9.08 \\
3 & 2.13 & 3.93 & 7.23 & 13.26 \\
\hline
\end{tabular}

Table 312 possible combined scores obtained from the prognostic index by combining one of three values for mitosis and one of four values for necrosis

\begin{tabular}{lllll}
\hline \multirow{5}{*}{ Mitosis } & \multicolumn{4}{l}{ Necrosis } \\
\cline { 2 - 5 } & 0 & 1 & 2 & 3 \\
\hline 1 & 0.38 & 0.99 & 1.60 & 2.21 \\
2 & 0.76 & 1.37 & 1.98 & 2.59 \\
3 & 1.14 & 1.75 & 2.36 & 2.97 \\
\hline
\end{tabular}

cases $)=0 \cdot 76-1 \cdot 14$, PIG $3(19$ cases $)=1 \cdot 37-$ 2.36, PIG 4 (13 cases) $=2 \cdot 59-2 \cdot 97$. The survival curves for the patients in each of these PIGs is shown in fig $\mathrm{lB}$.

Comparison of the new prognostic index and Bloom's method (fig 1) shows good separation of each of the tumour grades with the new grading system. The new prognostic index also identifies a group of patients with a distinctly poor prognosis. The five year survivals for each of the new PI grades is $78 \%$, $65 \%, 48 \%, 0 \%$ (grades $1-4$, respectively). In contrast, the five year survival rates for Bloom's grades $1-3$ are $82 \%, 60 \%, 40 \%$, respectively. The pie diagram (fig 2) illustrates what proportion of patients fall into each prognostic group for each of the methods.

\section{Discussion}

The aim of this study was to determine the prognostic importance of different features examined in histological sections of primary infiltrating breast carcinomas (not otherwise specified) and to ascertain their relative contribution to survival. This has permitted the formation of a simple prognostic index based on only two tumour features (necrosis and mitotic count).

In this new grading system patients are separated into four distinct groups-prognostic index grade PIG 1 (excellent prognosis); PIG 2 (good prognosis); PIG 3 (fair prognosis); PIG 4 (poor prognosis). In PIG 1 the five year survival is $78 \%$ and this is comparable

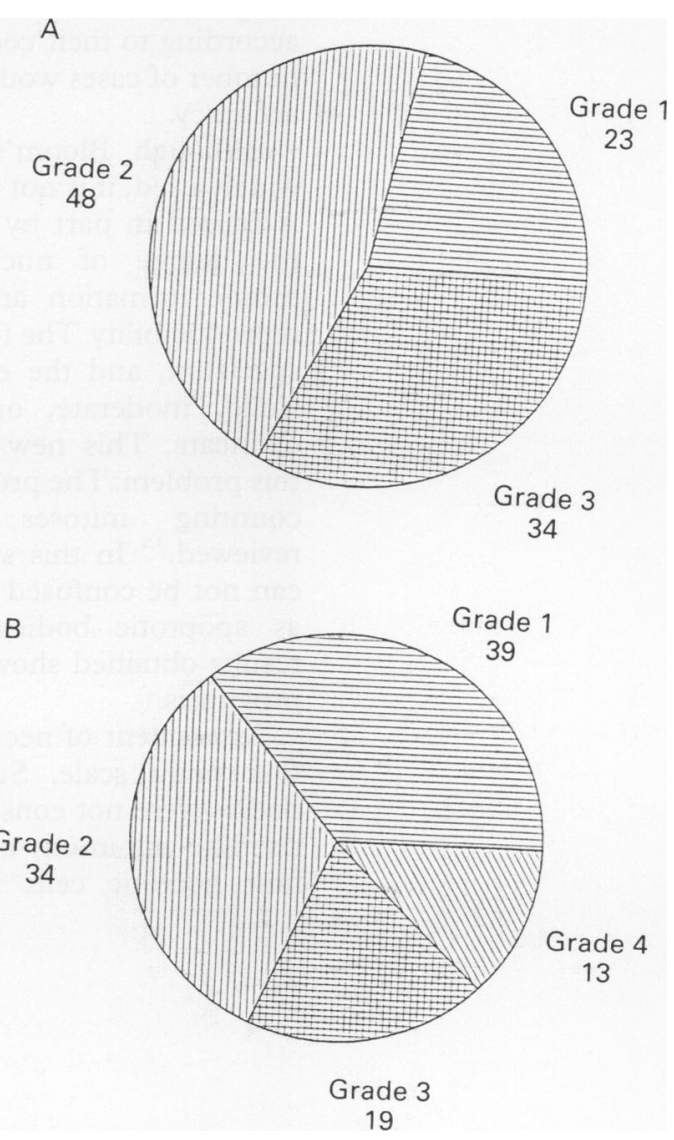

Figure 2 Pie chart showing proportion of cases in each grade (A) Bloom's grading, $(B)$ the new prognostic index. Numbers represent actual number of cases (total $=105)$. 
with that found in Bloom's grade 1 where survival is $82 \%$. In this new prognostic index patients with a poor outlook, unlike those with Bloom's grading method, are clearly separated. All patients in PIG 4 died after three years in contrast to patients with Bloom's grade 3 tumours where the five year survival was $40 \%$. One of the criticisms of Bloom's method is the fact that most patients fall into grade 2 and only a small proportion are identified as being in a good or poor prognostic group. This new method also separates patients with an intermediate outcome into those which can be considered a good prognosis and those with a fair prognosis.

The importance of mitosis counting in tumours is well recognised ${ }^{11}$ and the association between mitotic activity and breast cancer survival $^{1213}$ or recurrence ${ }^{1314}$ is well documented. Indeed, mitotic counts form the basis of Bloom's grading method. ${ }^{5}$ In this study statistical analysis of the three components (tubule formation, nuclear pleomorphism, and mitotic count) forming Bloom's grading, shows that mitotic count is the most significant parameter contributing to this index. This is consistent with the findings of Russo et al. ${ }^{13}$ Although the $t$ value for tubule formation and nuclear pleomorphism is $<1.96$, this does not necessarily mean that these factors have no effect on prognosis, but that the impact on prognosis is too small to be detected at the $5 \%$ significance level with the number of patients studied. The results do nevertheless suggest that if Bloom's method continues to be used, weighting of the individual components according to their coefficient based on a larger number of cases would increase the prognostic accuracy.

Although Bloom's method of grading is widely used, it is not universally accepted. This is caused in part by the difficulty in defining the degree of nuclear pleomorphism and tubule formation and inherent problems of reproducibility. The features span a continuous spectrum, and the exact points on the scale (mild, moderate, or good) are difficult to delineate. This new prognostic index avoids this problem. The problems encountered when counting mitoses have recently been reviewed. ${ }^{15}$ In this study only structures that can not be confused with other features, such as apoptotic bodies, are counted and the results obtained show a good correlation with prognosis.

Assessment of necrosis was performed on a four point scale. Scattered single apoptotic bodies were not considered evidence of necrosis. The minimum accepted was a cluster of four necrotic cells showing the features of either apoptosis or coagulative necrosis. Comedo type necrosis affecting cells within mammary ducts was ignored. No correlation was observed between histological grade and tumour necrosis (results not shown) and this is consistent with our previous findings. ${ }^{7}$

Fine needle aspiration biopsy is a potential cause of necrosis but we found that this was not a problem. When present it is easily recognised as a tract of loose vascular connective tissue containing plump fibroblasts. Evidence of haemorrhage (either free red cells or haemosiderin) is usually also present. Assessment of tumour fibrosis did not contribute any prognostic information.

As the choice of treatment modalities for breast cancer increase this new prognostic index should permit realistic stratification of patients and thus the most appropriate treatment. The use of only two variables may make this grading system more acceptable. Furthermore, the combination of this new prognostic index with tumour stage may allow even greater prognostic accuracy. A prospective study using this new prognostic index and to assess the reproducibility of this method is currently in progress.

1 Anderson TJ. Breast cancer screening: Principles and practicalities for histopathologists. In: Anthony PP, MacSween RNM, eds. Recent advances in Histopathology. 14. Edinburgh: Churchill Livingstone, 1989:43-61.

2 von Hansemann D. Die mikroskopische Diagnose der böesartigen Gerschwüelste. 2nd edn. Berlin: A Hirschwald, 1902:91-102.

3 Greenough RB. Varying degrees of malignancy in cancer of the breast. $¥$ Cancer Res 1925;9:453-63.

4 Patey DH, Scarff RW. The position of histology in the prognosis of carcinoma of the breast. Lancet 1928;i:801-4.

5 Bloom HJG, Richardson WW. Histological grading and prognosis in breast cancer. Br f Cancer 1957;11:359-77.

6 Elston CW. Grading of invasive carcinoma of the breast. In: Page DL, Anderson TJ, eds, Diagnostic histopatholoy of the breast. Edinburgh: Churchill Livingstone, 1988:300-11.

7 Parham DM, Robertson AJ, Brown RA. Morphometric analysis of breast carcinoma; association with survival. $f$ Clin Pathol 1988;41:173-7.

8 Fisher ER, Palekar AS, Gregorio RM, Redmond C, Fisher B. Pathological findings from the National Surgical B. Pathological findings from the National Surgical of tumour necrosis. Hum Pathol 1978;9:523-30.

9 Dixon JM, Page DL, Anderson TJ, et al. Long term survivors after breast cancer. Br f Surg 1985;72:445-8. 10 Cox DR. Regression models and life tables. $\mathcal{F} R$ Statist Soc B. 1972;34:187-220.

11 Baak JPA. Mitosis counting in tumours. Hum Pathol 1990;21:683-5.

12 Baak JPA, Dop HV, Kurver PHJ, Hermans J. The value of morphometry to classic prognosticators in breast cancer. Cancer 1985;56:374-82.

13 Russo J, Frederick J, Ownby HE, et al. Predictors of recurrence and survival of patients with breast cancer. $\mathrm{Am}$ recurrence and survival of patient

14 Stenkvist B, Bengtsson E, Dahlvist B, et al. Predicting breast cancer recurrence. Cancer 1982;50:2884-93.

15 Quinn CM, Wright NA. The clinical assessment of prouinn CM, Wright NA. The clinical assessment of pro-
liferation and growth in human tumours: evaluation of methods and applications as prognostic variables. $\mathcal{f}$ Clin Pathol 1990;16:93-102. 